\title{
Microbial Composition of Fermented Korean Soy Paste (Doenjang) Prepared by Adding Different Herbs during Fermentation
}

\author{
Mohamed Mannaa ${ }^{1,2}$, Seong-Soon $\mathrm{Cho}^{3}$, Young-Su Seo ${ }^{1, *}$ and Inmyoung Park ${ }^{3, *}$ \\ 1 Department of Integrated Biological Science, Pusan National University, Busan 46241, Korea; \\ mannaa@pusan.ac.kr \\ 2 Department of Plant Pathology, Cairo University, Giza 12613, Egypt \\ 3 School of Food and Culinary Arts, Youngsan University, Busan 48015, Korea; jss66m@ysu.ac.kr \\ * Correspondence: yseo2011@pusan.ac.kr (Y.-S.S.); inmpark@ysu.ac.kr (I.P.); \\ Tel.: +82-51-510-2267 (Y.-S.S.); +82-51-540-7236 (I.P.)
}

Citation: Mannaa, M.; Cho, S.-S.; Seo, Y.-S.; Park, I. Microbial Composition of Fermented Korean Soy Paste (Doenjang) Prepared by Adding Different Herbs during Fermentation. Fermentation 2021, 7, 93. https:// doi.org/10.3390/fermentation7020093

Academic Editors: Annalisa Serio and Clemencia Chaves-López

Received: 14 May 2021

Accepted: 8 June 2021

Published: 10 June 2021

Publisher's Note: MDPI stays neutral with regard to jurisdictional claims in published maps and institutional affiliations.

Copyright: (c) 2021 by the authors. Licensee MDPI, Basel, Switzerland. This article is an open access article distributed under the terms and conditions of the Creative Commons Attribution (CC BY) license (https:// creativecommons.org/licenses/by/ $4.0 /)$.

\begin{abstract}
The microbiota involved in Korean soy paste (doenjang) fermentation are the key factors determining its quality aspects. In this study, doenjang was prepared by adding three different herbs (Peppermint, Korean mint, and Coriander), and their effect on the microbiota composition was evaluated by $16 \mathrm{~S}$ rRNA metagenomic analyses. The $\beta$-diversity statistics indicated clear distinctions in the doenjang microbiota after the addition of herbs. A microbial composition analysis revealed that Tetragenococcus was among the dominant genera in the four doenjang groups, with a relatively higher abundance in the Korean mint group. In the Peppermint and Korean mint doenjang groups, the levels of undesirable microbes, such as opportunistic pathogens belonging to the genera Sphingobacterium and Pantoea, were significantly reduced. Additionally, other desirable microbes that are known to exhibit beneficial properties and produce bioactive compounds, such as Saccharopolyspora and Buttiauxella, were present at significantly higher levels. Significant negative correlations between members of the Bacillaceae and Halomonadaceae, Lactobacillaceae and Tissierellaceae, and the Lacobacillaceae and Erwiniaceae families were observed, indicating possible antagonistic relationships. Taken together, our results demonstrated that the incorporation of herbs, particularly Peppermint and Korean mint, during doenjang fermentation resulted in significant shifts in the microbial composition and could be utilized for beneficial effect on its fermentation.
\end{abstract}

Keywords: fermented soy paste; metagenomics analysis; coriander; peppermint; Korean mint

\section{Introduction}

Fermentation has been practiced for thousands of years for the preservation and storage of food materials [1]. Fermented foods are important sources of nutrition and have improved flavors; they are known for their potential health promoting and disease preventing properties, which contribute to their current worldwide popularity [2].

Doenjang is a traditional Korean fermented soy paste that has been consumed for thousands of years as a protein source and a basic seasoning ingredient. Traditionally, doenjang production is a two-stage process that is initiated by preparing meju, a dry fermented, moldy cooked soybean block, using naturally occurring bacteria and fungi. The second stage is performed by soaking the meju in salty water in porcelain containers under sunlight for several weeks to produce a solid portion that is called soybean paste (doenjang) and a liquid portion called soy sauce (gangjang). The doenjang is then mashed and further fermented before consumption through a few weeks to several years [3].

Doenjang has attracted much attention and popularity due to its health-related beneficial properties such as antioxidative, anti-inflammatory, immunity enhancing, and fibrinolytic activities [4-6]. Moreover, doenjang consumption may be associated with a 
lower incidence of type 2 diabetes, greater antimutagenic and anticancer activity, and an antiobesogenic effect [7-10]. The properties of doenjang including the health promotion effects and unique flavors and taste can be attributed to several bioactive compounds (e.g., bioactive peptides, isoflavones, saponins, phytic acid, and linoleic acid), produced as the result of the degradation and decomposition of soybean proteins and additional ingredients by the fermentation microbes [5,11,12]. Thereby, the microbes involved in this fermentation are the most critical components for understanding the phenomenon associated with the quality characteristics and safety aspects of doenjang $[13,14]$. The microbial composition of doenjang can be affected by several factors, mainly the environmental variables and the basic ingredients during fermentation [15].

Culture-dependent and culture-independent microbiological studies have attempted to reveal the microbial community structure in doenjang during fermentation. The culture-based methods indicated that members of the Bacillus genus, such as B. subtilis, B. licheniformis, and B. megaterium, were among the dominant bacteria, and members of the Aspergillus, Mucor, and Rhizopus genera were among the dominant fungi in doenjang and were considered to play important roles during fermentation $[11,16,17]$. The bacterial community of doenjang underwent a shift toward salt-tolerant bacteria as the fermentation of doenjang progressed, during which Bacillus spp., the lactic acid bacterium (LAB) Tetragenococcus halophilus, and coagulase-negative staphylococci were predominant [18]. However, these studies used culture-based methods and were limited in the detection and identification of the involved microbiota.

Culture-independent techniques such as 16S rRNA libraries and denaturing gradient gel electrophoresis (DGGE) have been used to study doenjang microbiota, and unlike in the case of previous studies, other microbes such as Staphylococcus equorum, Leuconostoc mesenteroides, T. halophilus, and Enterococcus faecium were found to be dominant $[19,20]$. More recently, an rRNA pyrosequencing study revealed that Bacillus spp., among bacteria, and Mucor spp., among fungi, were predominant in doenjang during the early fermentation period. However, as fermentation progressed, changes were observed in the microbial composition and bacteria such as Ignatzschineria, Myroides, Enterococcus, Corynebacterium, and Clostridium spp., and fungi such as Geotrichum, Scopulariopsis, Monascus, Fusarium, and Aspergillus spp. increased in abundance [21]. Another recent study showed that the phylum, Firmicutes, was the dominant taxon in both the traditional and modified doenjang samples; LAB, including mainly Tetragenococcus, Enterococcus, Leuconostoc, and Lactobacillus spp., were detected in most samples, whereas among the non-LAB, Staphylococcus and Ochrobactruma spp. were dominant [22]. In the same line, a recent study utilized an Illumina MiSeq platform to study the microbial composition of doenjang and revealed that members of the halophilic or halotolerant Tetragenococcus, Staphylococcus, Debaryomyces, Meyerozyma, Millerozyma, and Hyphopichia were predominant [23].

Herbs and spices have had a long history of use in foods as natural additives for improving health and sensory properties and are becoming more attractive as potential alternatives to pharmaceutical agents that provide significant protection against chronic diseases [24]. Various types of herbs such as Peppermint (Mentha piperita), Korean mint (Agastache rugosa), and Coriander (Coriandrum sativum) have been studied and are shown to be sources of a range of bioactive phytochemicals with a multitude of properties, including antimicrobial, antioxidant, and anticancer activities [25-27]. Hence, it is of great importance to investigate the role of different herbs and to demonstrate the positive effects of their incorporation in food.

A study on the microbes involved in doenjang fermentation and the effect of the ingredients used is crucial to understanding the fermentation process and the quality of the produced doenjang. Therefore, the objective of this study was to use high-throughput next generation sequencing-based metagenomics analyses to evaluate the effects of adding different herbs (Peppermint, Korean mint, and Coriander) on the microbial composition of doenjang. Controlling the process of doenjang fermentation by supplementation with dif- 
ferent ingredients may result in the establishment of customized amounts and compositions of ingredients with respect to the effects on the microbes involved in the fermentation.

\section{Materials and Methods}

\subsection{Preparation of Fermented Soy Paste Samples}

Fermented doenjang was prepared using the traditional Korean method under the guidance of a master craftsman of Korean cuisine at Youngsan University located in Pusan, Korea. Briefly, fermented doenjang-meju bricks (approximately 2 kg, Gigang County, Korea) were soaked in $6 \mathrm{~L}$ of a $20 \%(w / v)$ solar salt (Shinan, Korea) solution in a porcelain pot, and then 3 pieces of pure charcoal $(3 \mathrm{~cm} \times 3 \mathrm{~cm} \times 10 \mathrm{~cm})$ and 5 pieces of dried red pepper purchased from the local market in Gigang County were added. Each root-removed, trimmed herb with a weight equal to $10 \%$ of the meju weight was added. The mixture solution was stored in a sunny yard with no temperature control and the lid of the pot was opened by day and closed by night every day. After 45 days of fermentation, the mixture was separated into liquid (soy sauce) and solid parts (doenjang). Separated doenjang was mashed, homogenized, and continually stored under the same conditions, then samples were collected for metagenomic sequencing and analysis after 3 months of fermentation. Three different batches for each treatment and the control were prepared and used for the metagenomic analysis.

\subsection{Metagenomic DNA Extraction, Sequencing, and Bioinformatic Analysis}

The collected samples $(5 \mathrm{~g}$ ) were dissolved in $25 \mathrm{~mL}$ sterile distilled water, vigorously vortexed, filtered through double-layered sterilized cheesecloth to remove large particles, and centrifuged for $15 \mathrm{~min}(10,000 \times g)$ at $4{ }^{\circ} \mathrm{C}$. The collected pellet was weighed, and $250 \mathrm{mg}$ was used for metagenomic DNA extraction using a PowerSoil ${ }^{\circledR}$ DNA Isolation Kit (MO BIO Laboratories, Carlsbad, CA, USA), according to the manufacturer's protocol. The concentration and quality of the obtained metagenomic DNA were assessed using a NanoDrop2000 spectrophotometer (Thermo Fisher Scientific, Wilmington, DE, USA) and agarose gel electrophoresis. Samples were stored in Tris-EDTA buffer at $-20^{\circ} \mathrm{C}$ until use.

The metagenomics analysis was based on the sequencing of the V3 and V4 variable regions of the $16 \mathrm{~S}$ rRNA gene. PCR amplification, sequencing, and library preparation were carried out using the protocol provided along with the Herculase II fusion DNA polymerase Nextera XT Index Kit V2 on an Illumina ${ }^{\circledR}$ MiSeq ${ }^{\circledR}$ platform at Macrogen (Seoul, South Korea), using the following primer pair:

(F), 5'-TCGTCGGCAGCGTCAGATGTGTATAAGAGACAGCCTACGGGNGGCWGC AG-3';

(R), 5'-GTCTCGTGGGCTCGGAGATGTGTATAAGAGACAGGACTACHVGGGTAT CTAATCC- $3^{\prime}$.

The sequencing resulted in paired-end reads that were merged using the fast length adjustment of the short reads (FLASH; http://ccb.jhu.edu/software/FLASH/) [28]. The obtained raw sequences were purified and preprocessed to remove Illumina adaptors and short and low-quality reads using the Scythe (v0.994) (https://github.com/vsbuffalo/ scythe) and Sickle programs (https://github.com/najoshi/sickle). The clustering and annotation of the preprocessed sequences were performed using the CD-HIT-OTU-MiSeq and UCLUST algorithms, and the qualified sequences were arranged into the respective operational taxonomic units (OTUs) at a cut-off value of $97 \%$, using the Greengenes database (version 13_5) [29-31]. Microbiome analysis, including sequence annotation, diversity statistics, and taxonomic assignments of the obtained OTUs from the phylum to species level was carried out using the Quantitative Insights into Microbial Ecology version 2 (QIIME2) pipeline [32]. The obtained sequences were deposited as a sequence read archive in the National Center for Biotechnology Information database under the BioProject ID PRJNA628532. 


\subsection{Statistical Analysis}

Microbiome diversity and rarefaction were estimated using QIIME2 scripts, R (version 3.1.3), and the PAleontological STatistics software package (PAST) version 3.23 [33]. An analysis of variance (ANOVA) was performed using GLM procedures, and the means were separated using the least significant difference (LSD) test using by SAS (SAS Institute, Cary, NC, USA), wherein a $p$-value $<0.05$ was considered statistically significant. A Principal Coordinates Analysis (PCoA) was performed based on the Euclidean and Bray-Curtis distances. Pearson's correlation analysis and the plotting of correlograms representing the Pearson's correlation coefficient ( $\mathrm{r}$ ) matrices were performed using PAST.

\section{Results}

\subsection{Diversity of Fermented Soy Paste Microbiota}

The total read count in the next generation sequencing of the 12 doenjang samples (4 different samples in triplicate) was 1,558,143, with an average of 129,845 reads per sample. The total bases, read counts, GC\%, Q20\%, and Q30\% for each sample are shown in Table S1. After removing the low-quality reads and chimeras, 399,938 high-quality sequences were obtained, with an average of 33,328 \pm 4606 reads per sample (ranging from 26,522 to 40,950$)$.

The rarefaction analysis representing the number of OTUs with respect to the read counts of the sequences obtained from the doenjang samples showed partial levelling of the curves at around 1500 reads, which indicated that the sequencing was sufficient to represent the microbial composition, and that further sequencing would not result in a significant increase in the number of OTUs (Figure 1).

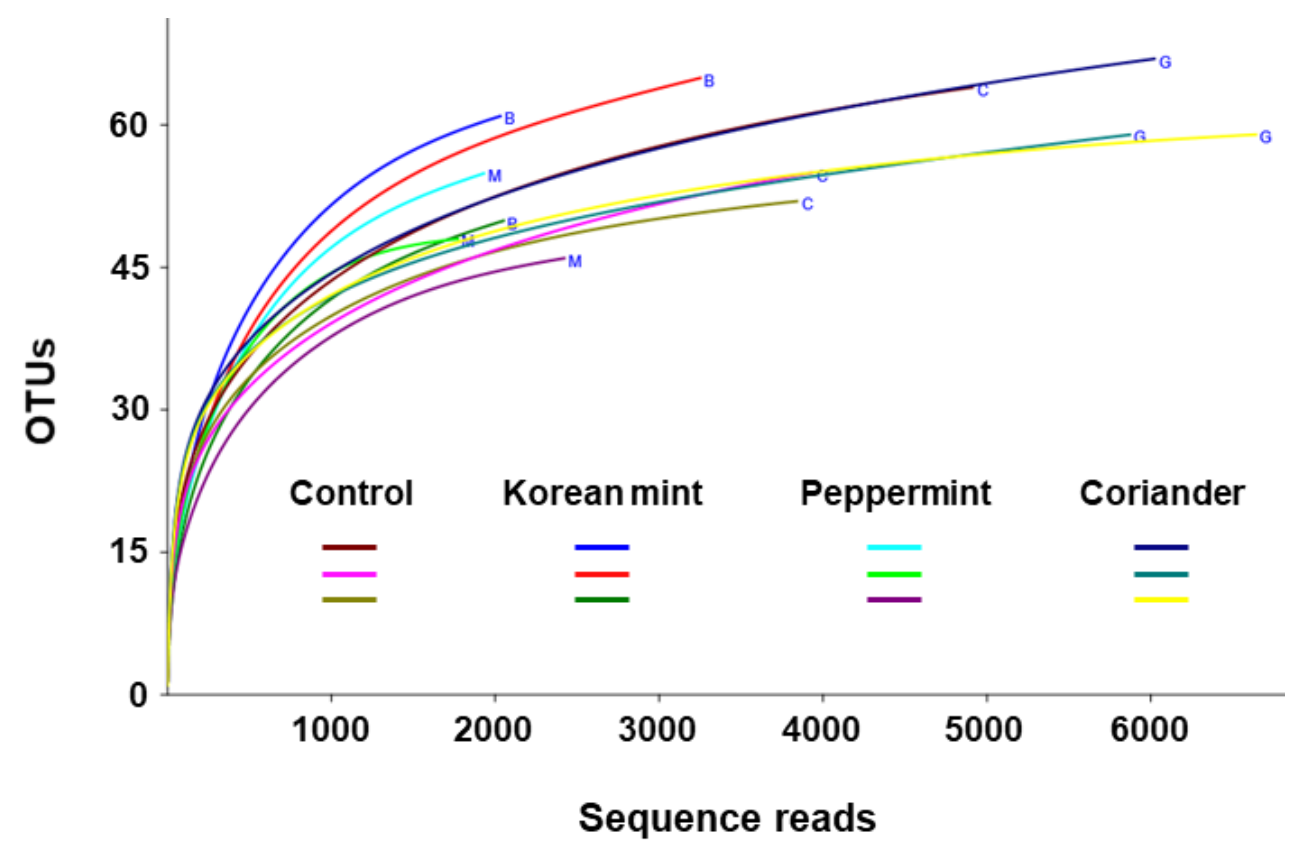

Figure 1. Rarefaction curve of $16 \mathrm{~S}$ rRNA sequence reads obtained against assigned operational taxonomic units (OTUs) to evaluate whether further sequencing may result in the detection of additional taxa in soy paste samples prepared by adding Peppermint, Korean mint, and Coriander with respect to that in control.

The doenjang samples supplemented with Korean mint exhibited higher richness compared to those supplemented with Peppermint or Coriander and the control; this was indicated by significantly $(p<0.05)$ higher OTUs and Chao1 indices (Figure 2A,C). Additionally, the Shannon diversity index was significantly $(p<0.05)$ lower for the doenjang samples supplemented with Peppermint and Korean mint, compared to that for the Co- 
riander and control groups (Figure 2B). The inverse Simpson index was significantly higher for the control doenjang group, compared to that for the other treatments (Figure 2D).

(A)

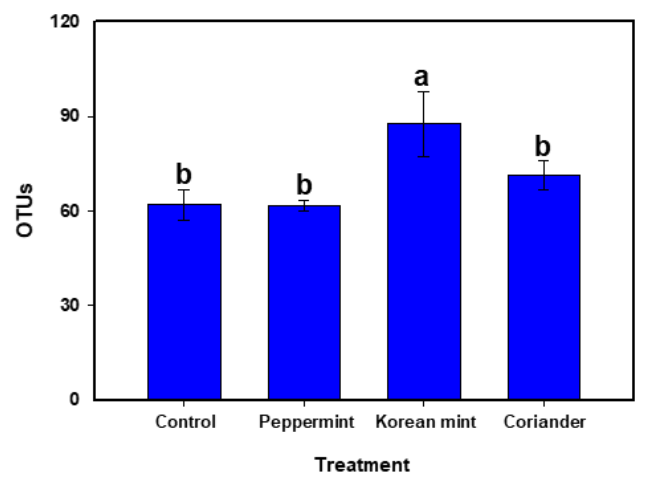

(C)

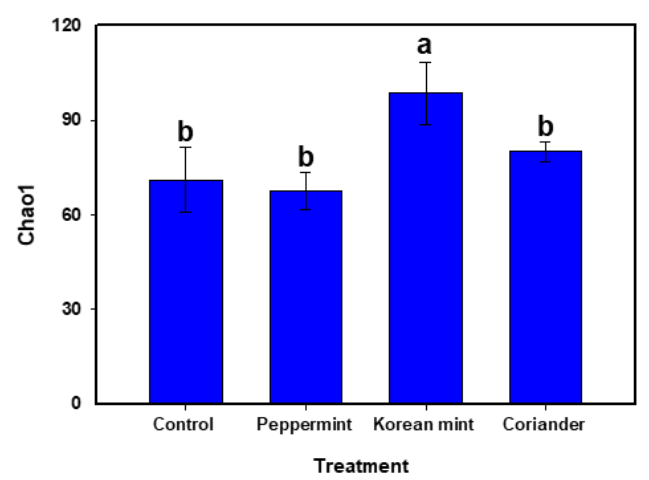

(B)

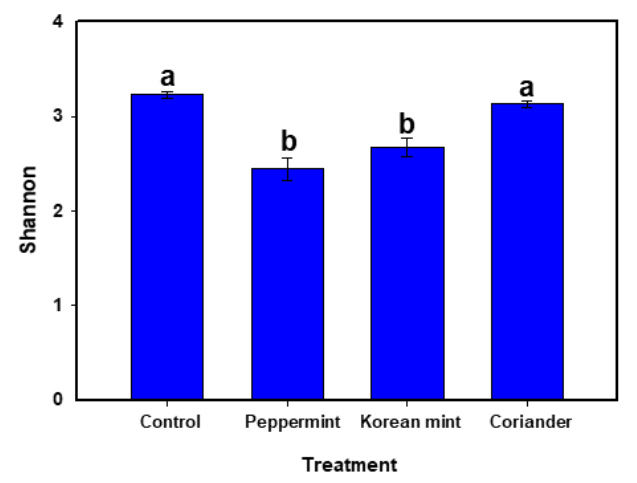

(D)

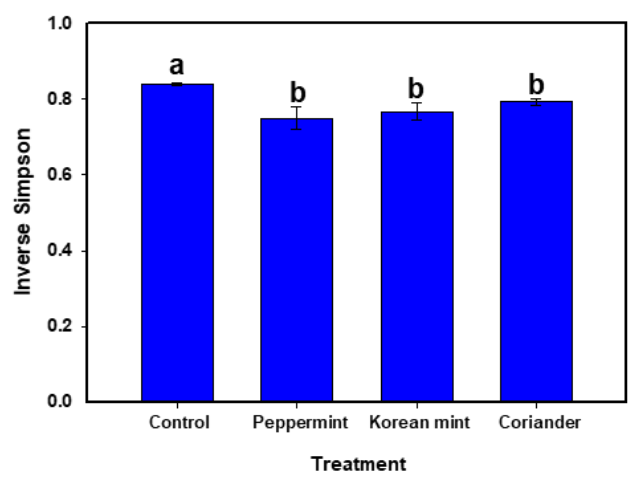

Figure 2. Alpha diversity in the soy paste samples prepared by adding Peppermint, Korean mint, and Coriander, relative to that in control. (A) Number of operational taxonomic units (OTUs), (B) Shannon diversity index, (C) Chao1 richness index, and (D) Inverse Simpson's index. Data are represented as the means \pm standard deviations in triplicate. Different letters on the error bars represent significant differences at $p<0.05$.

PCoA was performed using the Euclidean and UniFrac distances to illustrate the differences in relationship between the different doenjang samples. The different groups exhibited a clear distinction, with a close relationship between the Peppermint- and Koreanmint-supplemented doenjang groups based on the Euclidian distance and between the control and Coriander-supplemented doenjang groups, based on the UniFrac distance (Figure 3A,B).

\subsection{Effects of Peppermint, Korean Mint, and Coriander on Microbial Composition and Dominant Taxa in Doenjang}

The microbial community profiles of the different groups of doenjang showed noticeable differences at the taxonomic level (Figure 4). Four main phyla (Firmicutes, Proteobacteria, Actinobacteria, and Bacteroidetes) represented the bacterial profiles of the different doenjang samples. Bacteroidetes comprising mainly the genus Sphingobacterium were only found in significant levels in the control group, with an average relative abundance of $21.30 \%$; Actinobacteria comprising mainly the genus Saccharopolyspora were more abundant in the Peppermint doenjang, with an average abundance of $25.77 \%$ compared to $6.10 \%, 2.00 \%$, and $1.49 \%$ in the Korean mint, Coriander, and control doenjang, respectively (Figure $4 \mathrm{~A}$ ). 
(A)
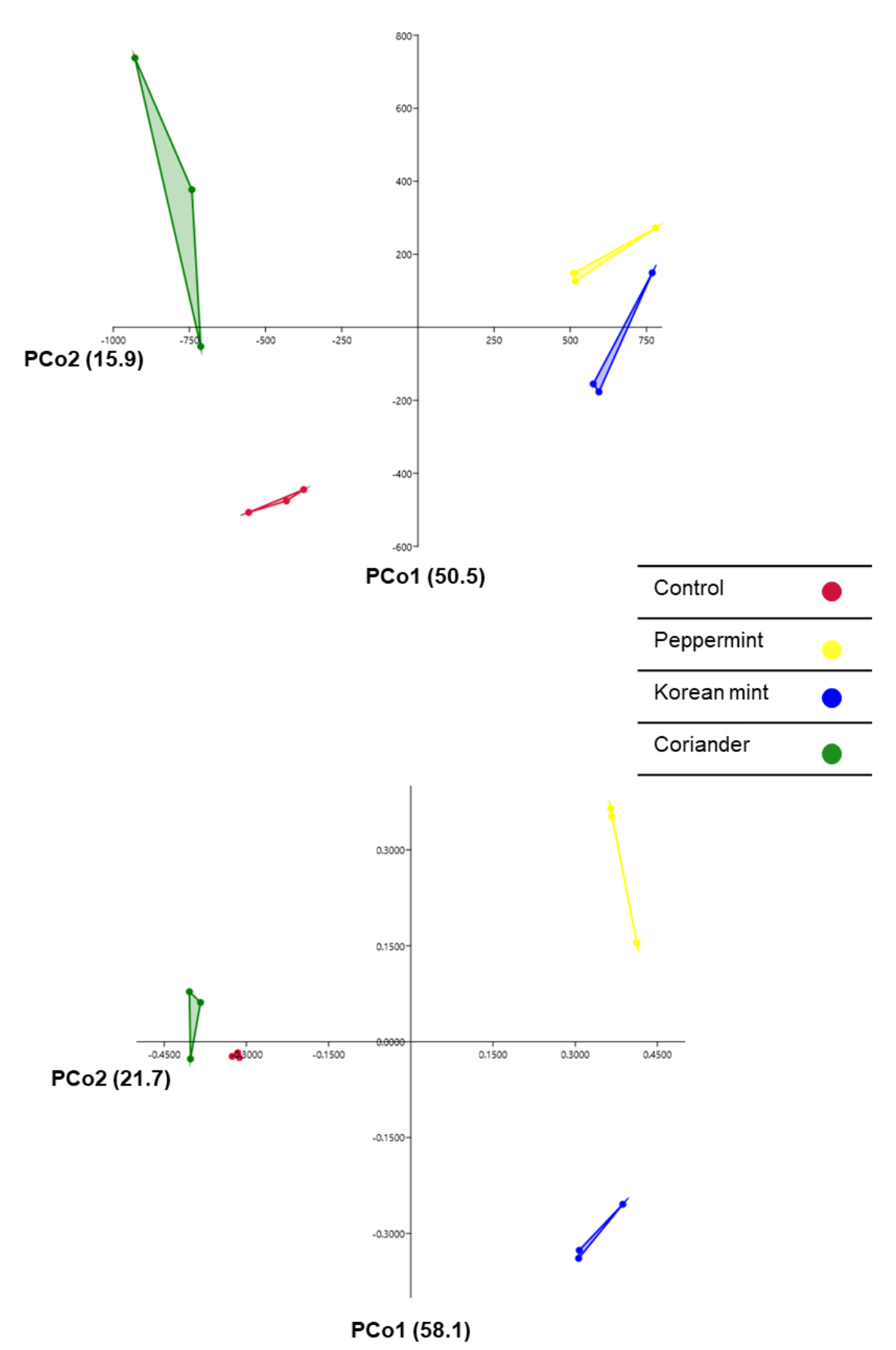

Figure 3. Principal Component Analysis (PCA) of (A) Euclidian and (B) Bray-Curtis distances of soy paste samples prepared by adding Peppermint, Korean mint, and Coriander, relative to those of the control. Each dot represents a replicate of the different groups of doenjang samples.

At the order level, Lactobacillales dominated the microbial communities in all the samples, with average relative abundances of $35.37 \%, 38.99 \%, 42.92 \%$, and $38.25 \%$ in the control, Peppermint, Korean mint, and Coriander doenjang groups, respectively. Sphingobacteriales were present in significant levels only in the control group, whereas Pseudonocardiales were only present in the Peppermint and Korean mint groups. Within the Proteobacteria, the ratio of Enterobacterales to Pseudomonadales was 18.79\%:12.23\%, 1.25\%:31.69\%, $31.28 \%: 18.87 \%$, and 35.16\%:5.11\% in the control, Peppermint, Korean mint, and Coriander doenjang groups, respectively. Moreover, Bacillales were present in higher abundance in the Coriander group, with an average relative abundance of $17.98 \%$ compared to $10.18 \%, 2.28 \%$, and $0.81 \%$ in the control, Peppermint, and Korean mint groups, respectively (Figure 4B). 
(A)

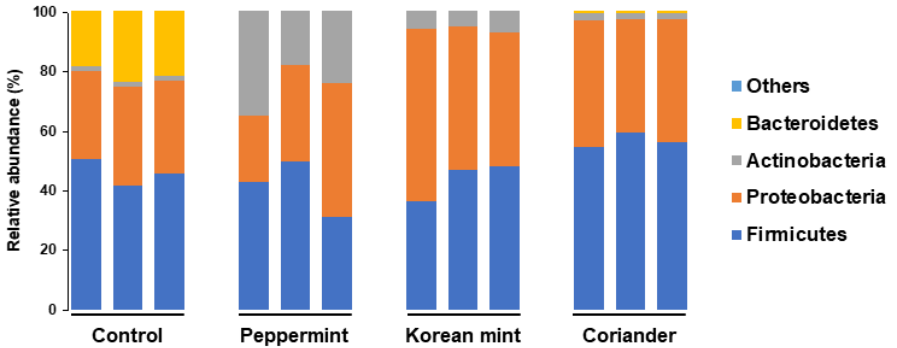

(B)

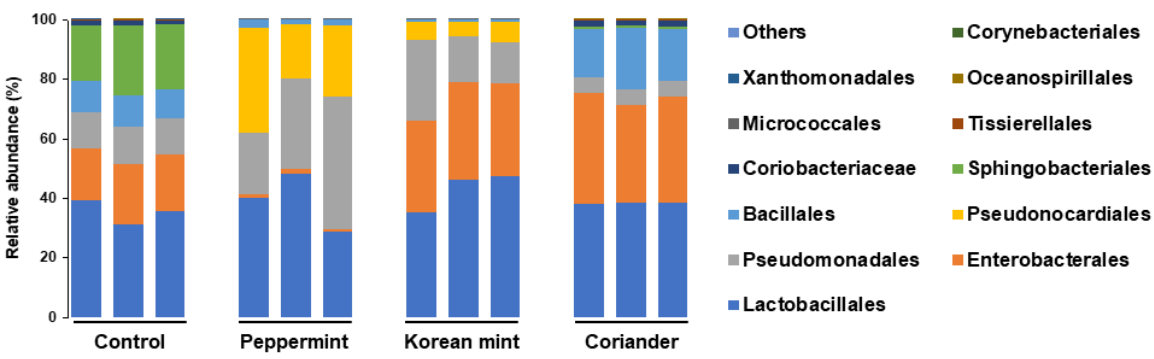

(C)

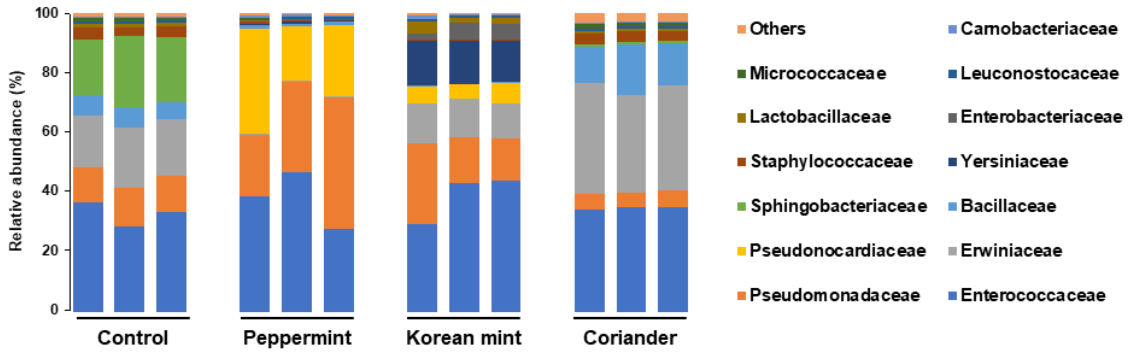

(D)
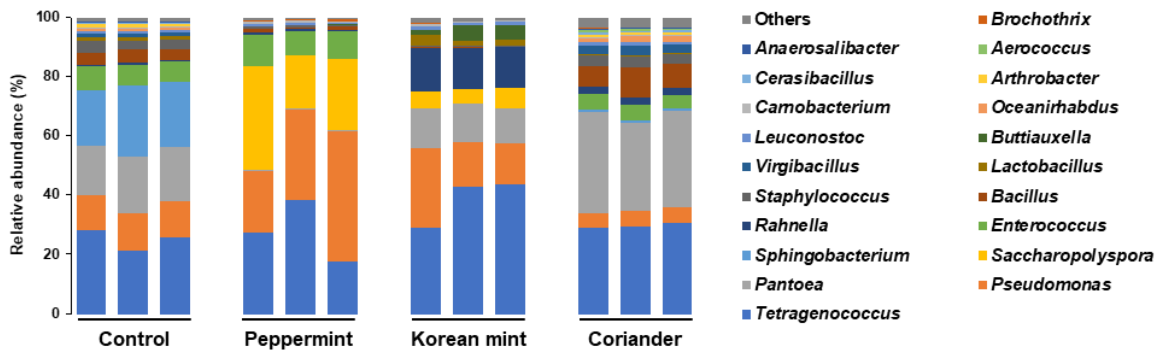

Figure 4. Stacked bar graphs representing the microbiome structure in soy paste samples prepared by adding Peppermint, Korean mint, and Coriander, relative to that of the control. (A) Phylum level, (B) order level, (C) family level, and (D) genus level.

At the family level, Enterococcaceae dominated the community in the control, Peppermint, and Korean mint doenjang samples, with average relative abundances of $32.83 \%$, $37.61 \%$, and $38.89 \%$, respectively. However, the Coriander group was dominated by Erwiniaceae followed by Enterococcaceae, with average relative abundances of $35.09 \%$ and $34.77 \%$, respectively. Erwiniaceae accounted for average relative abundances of $18.78 \%$, $0.41 \%$, and $12.63 \%$ in the control, Peppermint, and Korean mint doenjang groups, respectively. Pseudomonadaceae and Pseudonocardiaceae were present at higher levels in the Peppermint doenjang samples than in other groups, with average relative abundances of $31.64 \%$ and $25.56 \%$, respectively. Yersinia spp. were present at significant levels only in the Korean mint doenjang group, with an average relative abundance of $14.32 \%$, whereas Sphingobacteriaceae were found at significant levels only in the control group with an average relative abundance of $21.30 \%$ (Figure $4 \mathrm{C}$ ). 
At the genus level, Tetragenococcus was dominant in the control and Korean mint doenjang groups, accounting for average relative abundances of $25.40 \%$ and $38.81 \%$, respectively. The dominant genus in the Peppermint group was Pseudomonas, with an average relative abundance of $31.64 \%$ compared to $12.22 \%, 18.65 \%$, and $5.10 \%$ in the control, Korean mint, and Coriander doenjang groups, respectively. The genus Pantoea dominated the Coriander group, with an average relative abundance of $32.25 \%$ compared to $18.15 \%, 0.41 \%$, and $12.61 \%$ in the control, Peppermint, and Korean mint doenjang groups, respectively. Sphingobacterium spp. were the second most dominant species in the control group, with an average relative abundance of $21.30 \%$ and were present in minor levels in the other groups. Saccharopolyspora spp. were present at significant levels only in the Peppermint and Korean mint groups, with average relative abundances of $25.55 \%$ and $5.78 \%$, respectively (Figure 4D). The genus Rahnella was present at significant levels only in the Korean mint doenjang group, with an average relative abundance of $14.07 \%$ compared to $0.49 \%$, $0.66 \%$, and $2.60 \%$ in the control, Peppermint, and Coriander doenjang groups, respectively (Figure 4D).

\subsection{Correlation Analysis of Relative Abundance of Bacterial Families in Doenjang Samples}

Different significantly $(p<0.05)$ positive and negative correlations between the OTU counts corresponding to different bacterial families in all the tested doenjang samples $(n=12)$ were determined based on the Pearson's coefficient matrix. The strong positive and negative correlations indicated by the large blue and red circles (Figure 5) suggest the possible coexistence or antagonism between the different bacterial families. With respect to the doenjang fermentation microbiota, significant negative correlations between the Bacillaceae and Halomonadaceae, and the Lactobacillaceae, Microbacteriaceae, Micrococcaceae, and Tissierellaceae families were observed (Figure 5).

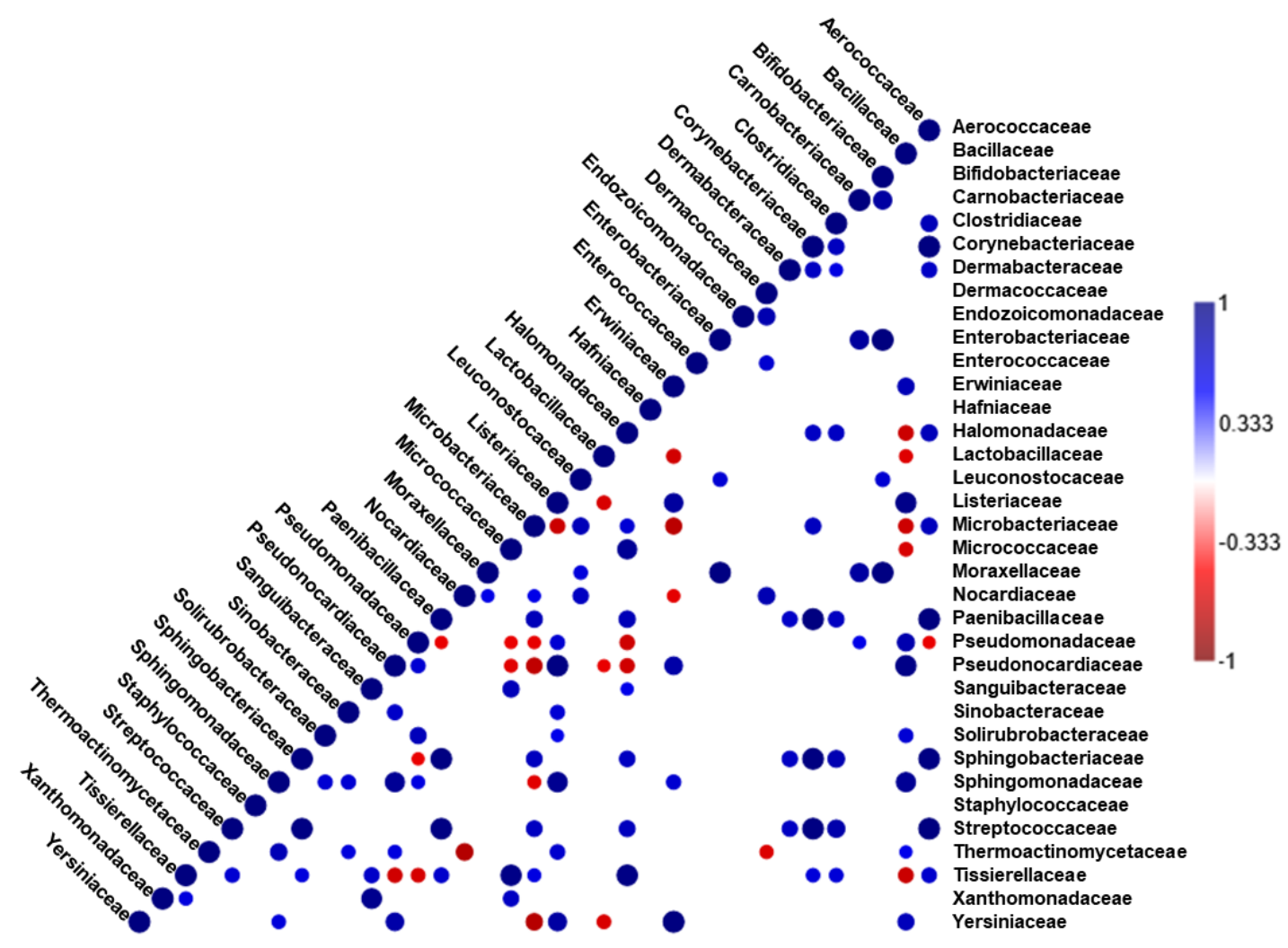

Figure 5. Correlogram representing the matrices of Pearson's correlation coefficient ( $r$ ) for different bacterial taxa (at family level) in soy paste samples $(n=12)$ prepared by adding Peppermint, Korean mint, and Coriander, relative to those for the control. Only significant $(p<0.05)$ positive (blue) and negative (red) correlations are shown in the graph. 


\section{Discussion}

The microbiota involved in doenjang fermentation are the key factors that determine the end-product's quality and safety aspects [13]. Therefore, it is important to study the microbial composition of doenjang, and to endeavor to provide fermentation conditions aimed at enhancing desirable microbes (e.g., microbes that produce bioactive metabolites and toxin-degrading enzymes contributing to better health), thereby reducing the levels of undesirable microbes such as those related to spoilage, or opportunistic human pathogens. Previous studies have shown that the ingredients used for doenjang preparation markedly affect the fermentation process and the properties of the end-product [34-37].

In this study, three different herbs (Peppermint, Korean mint, and Coriander) known to potentially produce various bioactive compounds with several beneficial properties, including the inhibition of harmful microbes [25-27] were used as supplements during doenjang fermentation, and their effects on the microbial composition of the prepared doenjang were evaluated using high-throughput sequencing-based metagenomics analyses. The microbial composition in the herb-supplemented doenjang showed noticeable shifts compared to that in the control; in particular, the Peppermint- and Korean-mint-supplemented doenjang showed greater shifts compared to the control and Coriander-supplemented doenjang. Generally, doenjang prepared with herbs showed an improved microbial composition as evidenced by an increase in the abundance of beneficial microbes and a reduction in potential spoilage and harmful microbes.

Basically, the conditions of doenjang fermentation induce a shift in the microbial composition toward an increase in the abundance of salt-tolerant microbes [18]. The halophilic LAB, Tetragenococcus, was among the dominant microbes in all the doenjang samples, with a relatively higher abundance in Korean mint supplemented doenjang than in the control. Jung et al. [13], functionally characterized the major microbial taxa responsible for fermentation, and concluded that although Bacillus and Enterococcus spp. were the dominant microbes, their abundance was not linked to the changes in the metabolite levels, whereas Tetragenococcus was linked to an increase in organic acids and a reduction in glucose and fructose levels; Lactobacillus was associated with an increase in $\gamma$-aminobutyric acid (GABA) and a decrease in galactose levels. Consistent with our results, T. halophilus has been shown to be among the predominant halophilic LAB during doenjang fermentation and produced desirable flavors in fermented soy products $[19,20,38]$.

Previous studies have also revealed several beneficial roles of T. halophilus in doenjang, such as antiallergy and aflatoxin degradation activity. The immunomodulatory activity of a strain of T. halophilus, which was selected for its ability to induce strong interleukin (IL)-12 production and T helper type 1 (Th1) immunity promotion, has been reported [39]. The oral administration of this strain was employed in another study to alleviate the severity of nasal symptoms in perennial allergic rhinitis [40]. Another strain of T. halophilus has been reported to degrade $92 \%$ of the aflatoxin B1 in a polluted fermented soy product [41].

In the same context, the level of Actinobacteria, which includes several members known to produce many bioactive compounds, was significantly higher in herb-supplemented doenjang, specifically in that prepared with Peppermint. In particular, the halophilic bacteria of the Saccharopolyspora genus were present in the Peppermint- and Korean-mintsupplemented doenjang, with a significantly high relative abundance; Saccharopolyspora are known producers of amylases, which transform starch into micromolecular sugars (such as glucose and mannose) that serve as carbon source precursors for the production of organic acids in addition to potential bioactive metabolites with anticancer and antimicrobial activities [42-44]. Moreover, halophilic members of the genus Buttiauxella, which were previously shown to produce antimicrobial metabolites active against several pathogenic and harmful bacteria and fungi, were present at relatively high abundances in the Koreanmint-supplemented doenjang and, to a lesser extent, in the Peppermint-supplemented doenjang but not in the control or the Coriander-supplemented doenjang [45].

However, the relative abundance of the phylum, Bacteroidetes, mainly composed of the genus Sphingomonas, was significantly low in the doenjang prepared with herbs; 
members belonging to the Sphingomonas genus are potentially harmful, and have been reported to infect humans $[46,47]$. The levels of other potentially harmful species belonging to the genus Pantoea, which are considered opportunistic pathogens, were significantly lower in the Peppermint-supplemented doenjang and high in the control and Coriandersupplemented doenjang [48].

The correlation analysis revealed specific relationships between different microbial taxa; interestingly, the Bacillaceae family showed a significant negative correlation with the halophilic and halotolerant Halomonadaceae, Lactobacillaceae, and Tissierellaceae families, which indicated that the levels of Bacillaceae could be reduced in the presence of such halophilic microbes. Moreover, Lactobacillaceae also showed a significant negative correlation with Erwiniaceae, which includes opportunistic pathogens belonging to the genus Pantoea, indicating that the high abundance of Lactobacillaceae played a role in the suppression of potentially harmful microbes.

It is noteworthy to mention that the bioinformatic analysis in this study was performed before the recent change in the taxonomy of Lactobacillus and the union of Lactobacillaceae and Leuconostocaceae families reported in Zheng et al. [49].

Taken together, our metagenomic analysis on doenjang prepared with different herbs revealed significant shifts in the microbial composition. Notably, the supplementation of doenjang with Peppermint and Korean mint during fermentation resulted in positive changes in the microbial composition with respect to the suppression of undesirable microbes and an increase in the abundance of beneficial microbes that have been previously shown to play key roles in doenjang fermentation and production of bioactive compounds. Further study is required to investigate the effect of adding herbs during doenjang fermentation for longer durations; additionally, metabolic profiling and sensory evaluation with respect to the microbial composition are also required.

Supplementary Materials: The following are available online at https:/ /www.mdpi.com/article/10 $.3390 /$ fermentation7020093/s1, Table S1: Sequencing results, showing the total bases, read count, $\mathrm{GC} \%, \mathrm{Q} 20 \%$, and Q30\% of each soy paste sample.

Author Contributions: I.P. and Y.-S.S. conceived the study and designed the experiments. M.M., S.-S.C. and I.P. performed the experiments and analyzed the data. M.M., Y.-S.S. and I.P. wrote the paper. All authors have read and agreed to the published version of the manuscript.

Funding: This work was supported by the Basic Science Research Program through the National Research Foundation of Korea (NRF) grant funded by the Ministry of Science, ICT and Future Planning (NRF-2018R1D1A1B07041112).

Institutional Review Board Statement: Not applicable.

Informed Consent Statement: Not applicable.

Data Availability Statement: Not applicable.

Conflicts of Interest: The authors declare no conflict of interest.

\section{References}

1. Cao, Z.-H.; Green-Johnson, J.M.; Buckley, N.D.; Lin, Q.-Y. Bioactivity of soy-based fermented foods: A review. Biotechnol. Adv. 2019, 37, 223-238. [CrossRef]

2. Rolle, R.; Satin, M. Basic requirements for the transfer of fermentation technologies to developing countries. Int. J. Food Microbiol. 2002, 75, 181-187. [CrossRef]

3. Lee, S.Y.; Lee, S.; Lee, S.; Oh, J.Y.; Jeon, E.J.; Ryu, H.S.; Lee, C.H. Primary and secondary metabolite profiling of doenjang, a fermented soybean paste during industrial processing. Food Chem. 2014, 165, 157-166. [CrossRef]

4. Kim, M.; Im, S.; Yoo, Y.; Kim, G.; Lee, J.H. Antioxidative materials in domestic Meju and Doenjang-(4)-Separation of phenolic compounds and their antioxidative activity. J. Korean Soc. Food Sci. Nutr. 1994, 23, 792-798.

5. Nam, Y.R.; Won, S.B.; Chung, Y.-S.; Kwak, C.S.; Kwon, Y.H. Inhibitory effects ofDoenjang, Korean traditional fermented soybean paste, on oxidative stress and inflammation in adipose tissue of mice fed a high-fat diet. Nutr. Res. Pr. 2015, 9, 235-241. [CrossRef]

6. Ra, K.; Oh, S.; Kim, J.; Suh, H.J. Isolation of fibrinolytic enzyme and $\beta$-glucosidase producing strains from Doenjang and op-timum conditions of enzyme production. J. Korean Soc. Food Sci. Nutr. 2004, 33, 439-442. 
7. Cha, Y.-S.; Yang, J.-A.; Back, H.-I.; Kim, S.-R.; Kim, M.-G.; Jung, S.-J.; O Song, W.; Chae, S.-W. Visceral fat and body weight are reduced in overweight adults by the supplementation ofDoenjang, a fermented soybean paste. Nutr. Res. Pract. 2012, 6, 520-526. [CrossRef]

8. Jung, K.-O.; Park, S.-Y.; Park, K.-Y. Longer aging time increases the anticancer and antimetastatic properties of doenjang. Nutrition 2006, 22, 539-545. [CrossRef] [PubMed]

9. Kwak, C.S.; Park, S.C.; Song, K.Y. Doenjang, a Fermented Soybean Paste, Decreased Visceral Fat Accumulation and Adipocyte Size in Rats Fed with High Fat Diet More Effectively Than Nonfermented Soybeans. J. Med. Food 2012, 15, 1-9. [CrossRef]

10. Kwon, D.Y.; Daily, J.W., III; Kim, H.J.; Park, S. Antidiabetic effects of fermented soybean products on type 2 diabetes. Nutr. Res. 2010, 30, 1-13. [CrossRef] [PubMed]

11. Kang, M.J.; Kim, S.H.; Joo, H.K.; Lee, G.S.; Yim, M.H. Isolation and identification of microorganisms producing the soy pro-teinhydrolyzing enzyme from traditional mejus. Appl. Biol. Chem. 2000, 43, 86-94.

12. Kwon, D.Y.; Hong, S.M.; Ahn, I.S.; Kim, M.J.; Yang, H.J.; Park, S. Isoflavonoids and peptides from meju, long-term fermented soybeans, increase insulin sensitivity and exert insulinotropic effects in vitro. Nutrition 2011, 27, 244-252. [CrossRef]

13. Jung, W.Y.; Jung, J.Y.; Lee, H.J.; Jeon, C.O. Functional Characterization of Bacterial Communities Responsible for Fermentation of Doenjang: A Traditional Korean Fermented Soybean Paste. Front. Microbiol. 2016, 7, 827. [CrossRef]

14. Park, S.; Song, K.-M.; Kim, H.-J.; Kim, B.-S.; Rhee, Y.K.; Lee, M.-K. Characterization and use of microbial communities in Doenjang to control the unpleasant odor of Ginkgo epicarp. Food Sci. Biotechnol. 2014, 23, 1959-1964. [CrossRef]

15. Park, H.-K.; Gil, B.; Kim, J.K. Characteristics of taste components of commercial soybean paste. Food Sci. Biotechnol. 2002, 11, 376-379.

16. Lee, S.S.; Park, K.H.; Choi, K.J.; Won, S. Identification and isolation of zygomycetous fungi found on maeju, a raw material of Korean traditional soysources. Korean J. Mycol. 1993, 21, 172-187.

17. Yoo, S.; Cho, W.; Kang, S.; Lee, S.H. Isolation and identification of microorganisms in Korean traditional soybena paste and soybean sauce. Korean J. Microbiol. Biotechnol. 1999, 27, 113-117.

18. Jeong, D.-W.; Kim, H.-R.; Jung, G.; Han, S.; Kim, C.-T.; Lee, J.-H. Bacterial community migration in the ripening of doenjang, a traditional Korean fermented soybean food. J. Microbiol. Biotechnol. 2014, 24, 648-660. [CrossRef]

19. Cho, K.M.; Seo, W.T. Bacterial diversity in a Korean traditional soybean fermented foods (doenjang and ganjang) by $16 \mathrm{~S}$ rRNA gene sequence analysis. Food Sci. Biotechnol. 2007, 16, 320-324.

20. Kim, T.-W.; Lee, J.-H.; Kim, S.-E.; Park, M.-H.; Chang, H.C.; Kim, H.-Y. Analysis of microbial communities in doenjang, a Korean fermented soybean paste, using nested PCR-denaturing gradient gel electrophoresis. Int. J. Food Microbiol. 2009, 131, 265-271. [CrossRef]

21. Jung, J.Y.; Lee, S.H.; Jeon, C.O. Microbial community dynamics during fermentation of doenjang-meju, traditional Korean fermented soybean. Int. J. Food Microbiol. 2014, 185, 112-120. [CrossRef]

22. Kim, M.J.; Kwak, H.S.; Jung, H.Y.; Kim, S.S. Microbial communities related to sensory attributes in Korean fermented soy bean paste (doenjang). Food Res. Int. 2016, 89, 724-732. [CrossRef]

23. Song, D.; Chun, B.; Lee, S.; Son, S.; Reddy, C.; Mun, H.; Jeon, C.; Lee, C. Comprehensive Metabolite Profiling and Microbial Communities of Doenjang (Fermented Soy Paste) and Ganjang (Fermented Soy Sauce): A Comparative Study. Foods 2021, 10, 641. [CrossRef]

24. Tapsell, L.C.; Hemphill, I.; Cobiac, L.; Sullivan, D.R.; Fenech, M.; Patch, C.S.; Roodenrys, S.; Keogh, J.B.; Clifton, P.; Williams, P.G.; et al. Health benefits of herbs and spices: The past, the present, the future. Med. J. Aust. 2006, 185, S1-S24. [CrossRef]

25. Areias, F.; Valentão, P.; Andrade, P.; Ferreres, F.; Seabra, R. Phenolic fingerprint of peppermint leaves. Food Chem. 2001, 73, 307-311. [CrossRef]

26. Park, C.H.; Yeo, H.J.; Baskar, T.B.; Park, Y.E.; Park, J.S.; Lee, S.Y.; Park, S.U. In Vitro Antioxidant and Antimicrobial Properties of Flower, Leaf, and Stem Extracts of Korean Mint. Antioxidants 2019, 8, 75. [CrossRef]

27. Wangensteen, H.; Samuelsen, A.B.; Malterud, K.E. Antioxidant activity in extracts from coriander. Food Chem. 2004, 88, $293-297$. [CrossRef]

28. Magoč, T.; Salzberg, S.L. FLASH: Fast Length Adjustment of Short Reads to Improve Genome Assemblies. Bioinformatics 2011, 27, 2957-2963. [CrossRef]

29. Edgar, R.C. Search and clustering orders of magnitude faster than BLAST. Bioinformatics 2010, 26, 2460-2461. [CrossRef]

30. McDonald, D.; Price, M.; Goodrich, J.; Nawrocki, E.P.; DeSantis, T.Z.; Probst, A.; Andersen, G.L.; Knight, R.; Hugenholtz, P. An improved Greengenes taxonomy with explicit ranks for ecological and evolutionary analyses of bacteria and archaea. ISME J. 2011, 6, 610-618. [CrossRef]

31. Li, W.; Chang, Y. CD-HIT-OTU-MiSeq, an improved approach for clustering and analyzing paired end MiSeq 16S rRNA sequences. BioRxiv 2017, 153783. [CrossRef]

32. Caporaso, J.G.; Kuczynski, J.; Stombaugh, J.; Bittinger, K.; Bushman, F.D.; Costello, E.K.; Fierer, N.; Peña, A.G.; Goodrich, J.K.; Gordon, J.I.; et al. QIIME Allows Analysis of High-Throughput Community Sequencing data. Nat. Methods 2010, 7, $335-336$. [CrossRef]

33. Hammer, O.; Harper, D.A.; Ryan, P.D. Palaeontological statistics software package for education and data analysis. Palaeontol. Electron. 2001, 4, 9 . 
34. Bahuguna, A.; Shukla, S.; Lee, J.S.; Bajpai, V.K.; Kim, S.-Y.; Huh, Y.S.; Han, Y.-K.; Kim, M. Garlic augments the functional and nutritional behavior of Doenjang, a traditional Korean fermented soybean paste. Sci. Rep. 2019, 9, 5436. [CrossRef]

35. Hwang, K.-M.; Jung, K.-O.; Song, C.-H.; Park, K.-Y. Increased Antimutagenic and Anticlastogenic Effects of Doenjang (Korean Fermented Soybean Paste) Prepared with Bamboo Salt. J. Med. Food 2008, 11, 717-722. [CrossRef]

36. Shim, J.M.; Lee, K.W.; Yao, Z.; Kim, H.-J.; Kim, J.H. Properties of Doenjang (Soybean Paste) Prepared with Different Types of Salts. J. Microbiol. Biotechnol. 2016, 26, 1533-1541. [CrossRef] [PubMed]

37. Shukla, S.; Lee, J.S.; Bajpai, V.K.; Khan, I.; Huh, Y.S.; Han, Y.-K.; Kim, M. Toxicological evaluation of lotus, ginkgo, and garlic tailored fermented Korean soybean paste (Doenjang) for biogenic amines, aflatoxins, and microbial hazards. Food Chem. Toxicol. 2019, 133, 110729. [CrossRef] [PubMed]

38. Yoo, S.K.; Kang, S.M.; Noh, Y.S. Quality properties on soy bean pastes made with microorganisms isolated from traditional soy bean pastes. Korean J. Microbiol. Biotechnol. 2000, 32, 1266-1270.

39. Masuda, S.; Yamaguchi, H.; Kurokawa, T.; Shirakami, T.; Tsuji, R.; Nishimura, I. Immunomodulatory effect of halophilic lactic acid bacterium Tetragenococcus halophilus Th221 from soy sauce moromi grown in high-salt medium. Int. J. Food Microbiol. 2008, 121, 245-252. [CrossRef]

40. Nishimura, I.; Igarashi, T.; Enomoto, T.; Dake, Y.; Okuno, Y.; Obata, A. Clinical Efficacy of Halophilic Lactic Acid Bacterium Tetragenococcus halophilus Th221 from Soy Sauce Moromi for Perennial Allergic Rhinitis. Allergol. Int. 2009, 58, 179-185. [CrossRef] [PubMed]

41. Li, J.; Huang, J.; Jin, Y.; Wu, C.; Shen, D.; Zhang, S.; Zhou, R. Aflatoxin B1 degradation by salt tolerant Tetragenococcus halophilus CGMCC 3792. Food Chem. Toxicol. 2018, 121, 430-436. [CrossRef]

42. Manivasagan, P.; Kang, K.-H.; Sivakumar, K.; Li-Chan, E.C.; Oh, H.-M.; Kim, S.-K. Marine actinobacteria: An important source of bioactive natural products. Environ. Toxicol. Pharmacol. 2014, 38, 172-188. [CrossRef]

43. Manivasagan, P.; Venkatesan, J.; Sivakumar, K.; Kim, S.K. Marine actinobacterial metabolites and their pharmaceutical po-tential. In Springer Handbook of Marine Biotechnology; Springer: Berlin/Heidelberg, Germany, 2015; pp. 1371-1386.

44. Nie, Z.; Zheng, Y.; Xie, S.; Zhang, X.; Song, J.; Xia, M.; Wang, M. Unraveling the correlation between microbiota succession and metabolite changes in traditional Shanxi aged vinegar. Sci. Rep. 2017, 7, 9240. [CrossRef]

45. Marzban, A.; Ebrahimipour, G.; Danesh, A. Bioactivity of a Novel Glycolipid Produced by a Halophilic Buttiauxella sp. and Improving Submerged Fermentation Using a Response Surface Method. Molecules 2016, 21, 1256. [CrossRef]

46. Lambiase, A.; Rossano, F.; Del Pezzo, M.; Raia, V.; Sepe, A.; De Gregorio, F.; Catania, M.R. Sphingobacterium respiratory tract infection in patients with cystic fibrosis. BMC Res. Notes 2009, 2, 262. [CrossRef]

47. Tronel, H.; Plesiat, P.; Ageron, E.; Grimont, P. Bacteremia caused by a novel species of Sphingobacterium. Clin. Microbiol. Infect. 2003, 9, 1242-1244. [CrossRef]

48. Cunningham, D.J.; Leber, A. Enterobacter, Cronobacter, and Pantoea Species. In Principles and Practice of Pediatric Infectious Diseases; Elsevier BV: Amsterdam, The Netherlands, 2018; pp. 824-827.e1.

49. Zheng, J.; Wittouck, S.; Salvetti, E.; Franz, C.M.A.P.; Harris, H.M.B.; Mattarelli, P.; O'Toole, P.W.; Pot, B.; Vandamme, P.; Walter, J.; et al. A taxonomic note on the genus Lactobacillus: Description of 23 novel genera, emended description of the genus Lactobacillus Beijerinck 1901, and union of Lactobacillaceae and Leuconostocaceae. Int. J. Syst. Evol. Microbiol. 2020, 70, 2782-2858. [CrossRef] 